\title{
Tight-and-Cheap Conic Relaxation for the AC Optimal Power Flow Problem
}

\author{
Christian Bingane, Student Member, IEEE, Miguel F. Anjos, Senior Member, IEEE and Sébastien Le Digabel
}

\begin{abstract}
The classical alternating current optimal power flow problem is highly nonconvex and generally hard to solve. Convex relaxations, in particular semidefinite, second-order cone, convex quadratic, and linear relaxations, have recently attracted significant interest. The semidefinite relaxation is the strongest among them and is exact for many cases. However, the computational efficiency for solving large-scale semidefinite optimization is lower than for second-order cone optimization. We propose a conic relaxation obtained by combining semidefinite optimization with the reformulation-linearization technique, commonly known as RLT. The proposed relaxation is stronger than the second-order cone relaxation and nearly as tight as the standard semidefinite relaxation. Computational experiments using standard test cases with up to 6515 buses show that the time to solve the new conic relaxation is up to one order of magnitude lower than for the chordal relaxation, a semidefinite relaxation technique that exploits the sparsity of power networks.
\end{abstract}

Index Terms-Conic optimization, optimal power flow, power systems, semidefinite programming.

\section{NOMENCLATURE}

\section{A. Notations}

$\mathbb{R} / \mathbb{C}$

$\mathbb{H}^{n}$

$\mathrm{j}$

$a / \mathrm{a}$

a/a

A/A

Set of real/complex numbers,

Set of $n \times n$ Hermitian matrices,

Imaginary unit,

Real/complex number,

Real/complex vector,

Real/complex matrix.

\section{B. Operators}

$\operatorname{Re}(\cdot) / \operatorname{Im}(\cdot)$

$(\cdot)^{*}$

$|\cdot|$

$\angle(\cdot)$

$(\cdot)^{T}$

$(\cdot)^{H}$

$\operatorname{rank}(\cdot)$

Real/imaginary part operator,

Conjugate operator,

Magnitude or cardinality set operator,

Phase operator,

Transpose operator,

Conjugate transpose operator,

Rank operator.

\section{Input data}

$\mathscr{P}=(\mathcal{N}, \mathcal{L}) \quad$ Power network,

$\mathcal{N} \quad$ Set of buses,

$\mathcal{G}=\bigcup_{k \in \mathcal{N}} \mathcal{G}_{k} \quad$ Set of generators,

The authors are with the Department of Mathematics and Industrial Engineering, Polytechnique Montreal, Montreal, Quebec, Canada H3C 3A7; and the GERAD research center, Montreal, Quebec, Canada H3T 2A7. Emails: christian.bingane@polymtl.ca, anjos@stanfordalumni.org, sebastien.ledigabel@polymtl.ca.

This research was supported by the NSERC-Hydro-Quebec-Schneider Electric Industrial Research Chair.
$\mathcal{G}_{k}$

$\mathcal{L}$

$p_{D k} / q_{D k}$

$g_{k}^{\prime} / b_{k}^{\prime}$

$c_{g 2}, c_{g 1}, c_{g 0}$

$\mathrm{y}_{\ell}^{-1}=r_{\ell}+\mathrm{j} x_{\ell}$

$b_{\ell}^{\prime}$

$t_{\ell}$

\section{Variables}

$p_{G g} / q_{G g}$

$\mathrm{v}_{k}$

$p_{f \ell} / q_{f \ell}$

$p_{t \ell} / q_{t \ell}$

$\mathbf{T}$ I. INTRODUCTION HE optimal power flow (OPF) problem, introduced by Carpentier in 1962 [1], seeks to find a network operating point that optimizes an objective function such as generation cost subject to power flow equations and other operational constraints. A broad survey of the history of the problem and the related optimization methods appears in [2]-[4].

According to [3], the general OPF problem may be modelled using linear, mixed-integer linear, nonlinear, or mixed integer nonlinear optimization. We focus on the nonlinear version, also called alternating current optimal power flow (ACOPF) problem. The ACOPF problem is nonconvex and NP-hard [5], [6]. One way to tackle it is to use convex relaxations of the nonconvex constraints.

A conic optimization problem is a class of convex optimization problem that consists in optimizing a linear function over the intersection of an affine subspace and a convex cone. When the cone is the nonnegative orthant, the second-order cone, or the positive semidefinite matrices set, the conic optimization problem is a linear, a second-order cone or a semidefinite program respectively. A large theory can be found in [7] on convex optimization or in [8] on semidefinite optimization.

Since the ACOPF problem can be cast as a quadratically constrained quadratic program (QCQP), two principal conic relaxations have been proposed in the last decade: the second-order cone programming (SOCP) relaxation [9] and the semidefinite programming (SDP) relaxation [10]. These two relaxations offer several advantages. First, they can lead 
to global optimality. Second, because they are relaxations, they provide a bound on the global optimal value of the ACOPF problem. Third, if one of them is infeasible, then the ACOPF problem is infeasible.

We should note that the SDP relaxation is stronger than the SOCP relaxation but requires heavier computation. Therefore, a chordal relaxation was proposed in [11] in order to exploit the fact that power networks are not densely connected, thus reducing data storage and increasing computation speed. A full literature review on these three relaxations can be found in [12], [13]. Other convex relaxations have been developed in $[14]-[18]$.

For radial networks, the SOCP relaxation is tantamount to the SDP relaxation. In this case, one would normally solve the first one rather than the second one due to the difference in computation time. For general meshed networks, it would be interesting to develop a relaxation as fast as the SOCP relaxation and as strong as the SDP relaxation. For example, three strong SOCP relaxations were developed in [19] that are very close in quality to the SDP relaxation and are faster to solve.

In this paper, we present a new conic relaxation that offers a favourable trade-off between the SOCP and the SDP relaxations for large-scale instances of ACOPF in terms of optimality gap and computation time. This relaxation is obtained through a combination of semidefinite optimization and the reformulation-linearization technique, known as RLT.

The remainder of this paper is organized as follows. In Section II, we define the mathematical model of the ACOPF problem (without loss of generality). In Section III, we describe principal conic relaxations of the ACOPF problem, especially semidefinite and second-order cone relaxations. In Section IV, we present the new conic relaxation, and we present computational results in Section V. Section VI concludes the paper.

\section{ACOPF: FORMULATION}

Consider a typical power network $\mathscr{P}=(\mathcal{N}, \mathcal{L})$, where $\mathcal{N}=$ $\{1, \ldots, n\}$ and $\mathcal{L} \subseteq \mathcal{N} \times \mathcal{N}$ denote respectively the set of buses and the set of branches (transmission lines, transformers and phase shifters). Each branch $\ell \in \mathcal{L}$ has a from end $k$ (on the tap side) and a to end $m$ as modeled in [20]. We note $\ell=(k, m)$. The ACOPF problem is given as:

$$
\min \sum_{g \in \mathcal{G}} c_{g 2} p_{G g}^{2}+c_{g 1} p_{G g}+c_{g 0}
$$

over variables $\boldsymbol{p}_{G}, \boldsymbol{q}_{G} \in \mathbb{R}^{|\mathcal{G}|}, \boldsymbol{p}_{f}, \boldsymbol{q}_{f}, \boldsymbol{p}_{t}, \boldsymbol{q}_{t} \in \mathbb{R}^{|\mathcal{L}|}$, and $\mathbf{v} \in \mathbb{C}^{|\mathcal{N}|}$, subject to

- Power balance equations:

$$
\begin{aligned}
& \sum_{g \in \mathcal{G}_{k}} p_{G g}-p_{D k}-g_{k}^{\prime}\left|\mathrm{v}_{k}\right|^{2}= \\
& \sum_{\ell=(k, m) \in \mathcal{L}} p_{f \ell}+\sum_{\ell=(m, k) \in \mathcal{L}} p_{t \ell} \forall k \in \mathcal{N}, \\
& \sum_{g \in \mathcal{G}_{k}} q_{G g}-q_{D k}+b_{k}^{\prime}\left|\mathrm{v}_{k}\right|^{2}=
\end{aligned}
$$

$$
\sum_{\ell=(k, m) \in \mathcal{L}} q_{f \ell}+\sum_{\ell=(m, k) \in \mathcal{L}} q_{t \ell} \forall k \in \mathcal{N},
$$

- Line flow equations:

$$
\begin{aligned}
\frac{\mathrm{v}_{k}}{\mathrm{t}_{\ell}} & {\left[\left(\mathrm{j} \frac{b_{\ell}^{\prime}}{2}+\mathrm{y}_{\ell}\right) \frac{\mathrm{v}_{k}}{\mathrm{t}_{\ell}}-\mathrm{y}_{\ell} \mathrm{v}_{m}\right]^{*} } \\
& =p_{f \ell}+\mathrm{j} q_{f \ell} \forall \ell=(k, m) \in \mathcal{L}, \\
\mathrm{v}_{m} & {\left[-\mathrm{y}_{\ell} \frac{\mathrm{v}_{k}}{\mathrm{t}_{\ell}}+\left(\mathrm{j} \frac{b_{\ell}^{\prime}}{2}+\mathrm{y}_{\ell}\right) \mathrm{v}_{m}\right]^{*} } \\
& =p_{t \ell}+\mathrm{j} q_{t \ell} \forall \ell=(k, m) \in \mathcal{L},
\end{aligned}
$$

- Generator power capacities:

$$
\underline{p}_{G g} \leq p_{G g} \leq \bar{p}_{G g}, \underline{q}_{G g} \leq q_{G g} \leq \bar{q}_{G g} \forall g \in \mathcal{G},
$$

- Line thermal limits:

$$
\left|p_{f \ell}+\mathrm{j} q_{f \ell}\right| \leq \bar{s}_{\ell},\left|p_{t \ell}+\mathrm{j} q_{t \ell}\right| \leq \bar{s}_{\ell} \forall \ell \in \mathcal{L},
$$

- Voltage magnitude limits:

$$
\underline{v}_{k} \leq\left|\mathrm{v}_{k}\right| \leq \bar{v}_{k} \forall k \in \mathcal{N}
$$

- Reference bus constraint:

$$
\angle \mathrm{v}_{1}=0 .
$$

The objective function (1a) is the cost of conventional generation as commonly used in the literature. Constraints (1b)-(1e) are derived from Kirchhoff's laws and represent power flows in the network. Constraint (1i) specifies bus $k=1$ as the reference bus. We assume that $\underline{v}_{k}>0$ for all $k \in \mathcal{N}$ in (1h), and that generation cost $c_{g 2} p_{G g}^{2}+c_{g 1} p_{G g}+c_{g 0}$ is a convex function for all $g \in \mathcal{G}$.

Due to the nonconvex constraints (1d)-(1e), (1) is highly nonconvex and NP-hard [5], [6]. Applying local methods to this problem provides no guarantee on the optimality of any solution found. Moreover, it is intractable to solve to global optimality for large-scale instances.

\section{ACOPF: CONIC RELAXATIONS}

\section{A. Semidefinite relaxation}

With $\mathrm{V}=\mathbf{v} \mathbf{v}^{H}$, the ACOPF problem (1) can be reformulated as follows

$$
\begin{aligned}
& \min (1 \mathrm{a}) \\
& \text { s.t. }(1 \mathrm{f}),(1 \mathrm{~g}),(1 \mathrm{i}), \\
& \sum_{g \in \mathcal{G}_{k}} p_{G g}-p_{D k}-g_{k}^{\prime} \mathrm{V}_{k k}= \\
& \sum_{\ell=(k, m) \in \mathcal{L}} p_{f \ell}+\sum_{\ell=(m, k) \in \mathcal{L}} p_{t \ell} \forall k \in \mathcal{N}, \\
& \sum_{g \in \mathcal{G}_{k}} q_{G g}-q_{D k}+b_{k}^{\prime} \mathrm{V}_{k k}= \\
& \sum_{\ell=(k, m) \in \mathcal{L}} q_{f \ell}+\sum_{\ell=(m, k) \in \mathcal{L}} q_{t \ell} \forall k \in \mathcal{N}, \\
& \quad \frac{\left.\mathrm{y}_{\ell}\right|^{2}}{\mid \mathrm{t}^{2}}\left(-\mathrm{j} \frac{b_{\ell}^{\prime}}{2}+\mathrm{y}_{\ell}^{*}\right) \mathrm{V}_{k k}-\frac{\mathrm{y}_{\ell}}{\mathrm{t}_{\ell}} \mathrm{V}_{k m} \\
& =p_{f \ell}+\mathrm{j} q_{f \ell} \forall \ell=(k, m) \in \mathcal{L},
\end{aligned}
$$




$$
\begin{aligned}
& -\frac{\mathrm{y}_{\ell}^{*}}{\mathrm{t}_{\ell}^{*}} \mathrm{~V}_{m k}+\left(-\mathrm{j} \frac{b_{\ell}^{\prime}}{2}+\mathrm{y}_{\ell}^{*}\right) \mathrm{V}_{m m} \\
& =p_{t \ell}+\mathrm{j} q_{t \ell} \forall \ell=(k, m) \in \mathcal{L}, \\
& \underline{v}_{k}^{2} \leq \mathrm{V}_{k k} \leq \bar{v}_{k}^{2} \forall k \in \mathcal{N}, \\
& \mathrm{V}=\mathbf{v} \mathbf{v}^{H}
\end{aligned}
$$

The nonconvexity of (2) is captured by the constraint (2f). We can show that $\mathrm{V}=\mathbf{v} \mathbf{v}^{H}$ if and only if $\mathrm{V} \succeq 0$ and $\operatorname{rank}(\mathrm{V})=1$. The semidefinite relaxation (SDR) in Model 1 is obtained by dropping the rank constraint. It was first introduced in [10] and later, a dual relaxation was developed in [21].

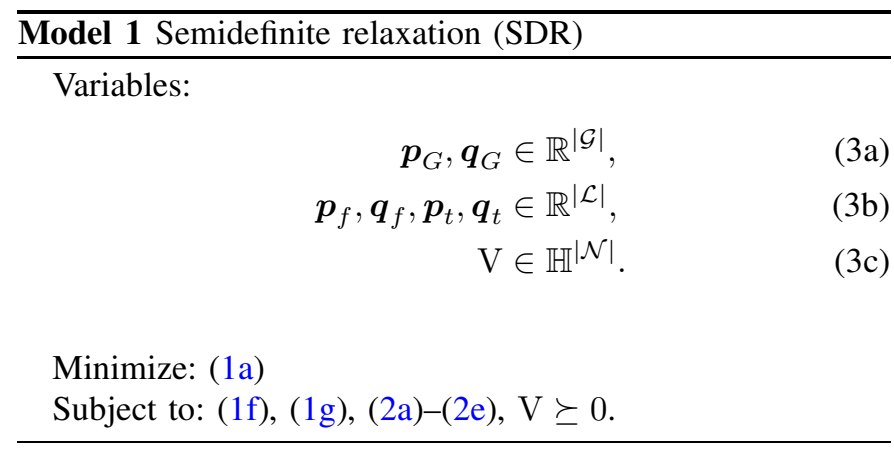

If the optimal solution $\hat{\mathrm{V}}$ of SDR is a rank-one matrix, then there exists a complex vector $\hat{\mathbf{v}}$, global optimal solution of (1). In the literature, there are numerous examples where SDR is exact. However, its exactness is only guaranteed for a few classes of problems under some assumptions [13].

On the other hand, solving SDR for large-scale power systems (more than a thousand of buses) is computationally very expensive. In order to reduce data storage and increase computational speed, [11] proposes to exploit in SDR the sparsity of the OPF problem. This methodology, as we explain in Section III-B, suggests to replace the positive semidefinite matrix $\mathrm{V}$ by less-sized positive semidefinite submatrices defined on a chordal extension of the power network [11], [12], [19].

\section{B. Chordal relaxation}

Let us interpret the network $\mathscr{P}=(\mathcal{N}, \mathcal{L})$ as a connected, simple and undirected graph $\mathscr{G}=(\mathcal{N}, \mathcal{E})$ where $\mathcal{N}=\{1, \ldots, n\}$ represents the set of vertices and $\mathcal{E}=$ $\{\{k, m\}:(k, m)$ or $(m, k) \in \mathcal{L}\}$, the set of edges. The power flow equations (1b)-(1e) in (1) depend only on $\mathrm{V}_{k k}:=\left|\mathrm{v}_{k}\right|^{2}$, $k \in \mathcal{N}$, and $\mathrm{V}_{k m}:=\mathrm{v}_{k} \mathrm{v}_{m}^{*},\{k, m\} \in \mathcal{E}$. In other words, except for the constraint $\mathrm{V} \succeq 0$, SDR depends only on a partial matrix $\mathrm{V}_{\mathscr{G}}$. A partial matrix means a matrix in which only some of the entries are specified [12], [22].

A subset $\mathcal{K} \subseteq \mathcal{N}$ is a clique if every two distinct vertices in $\mathcal{K}$ are adjacent in $\mathscr{G}$. A clique $\mathcal{K}$ is maximal in $\mathscr{G}$ if it is not a subset of a larger clique $\mathcal{K}^{\prime}$. A cycle is a sequence $k_{1}-k_{2}-\ldots-k_{\gamma}-k_{1}$ of $\gamma$ distinct vertices such that $\left\{k_{1}, k_{2}\right\},\left\{k_{2}, k_{3}\right\}, \ldots,\left\{k_{\gamma-1}, k_{\gamma}\right\},\left\{k_{\gamma}, k_{1}\right\} \in \mathcal{E}$, where $\gamma \geq 3$ is the length of the cycle. A chord of a cycle $k_{1}-k_{2}-\ldots-k_{\gamma}-k_{1}$ is an edge $\left\{k_{i}, k_{j}\right\} \in \mathcal{E}$ such that $1 \leq i<j \leq \gamma$ and $2 \leq j-i \leq \gamma-2$.
$\mathscr{G}$ is chordal if every cycle of 4 and more vertices has a chord. A chordal extension of $\mathscr{G}$ is a chordal graph $\mathscr{G}^{\prime}=$ $\left(\mathcal{N}, \mathcal{E}^{\prime}\right)$ that contains $\mathscr{G}$, i.e. $\mathcal{E} \subseteq \mathcal{E}^{\prime}$. It was proved in [22] that the constraint $\mathrm{V} \succeq 0$ in SDR is equivalent to $\mathrm{V}_{\mathcal{K}} \succeq 0$ for every maximal clique $\mathcal{K}$ of a chordal extension $\mathscr{G}^{\prime}$ of $\mathscr{G}$. $\mathrm{V}_{\mathcal{K}}$ is the submatrix of $\mathrm{V}$ in which the set of row indices that remain and the set of column indices that remain are both $\mathcal{K}$. Thus, the chordal relaxation (CHR) is given in Model 2.

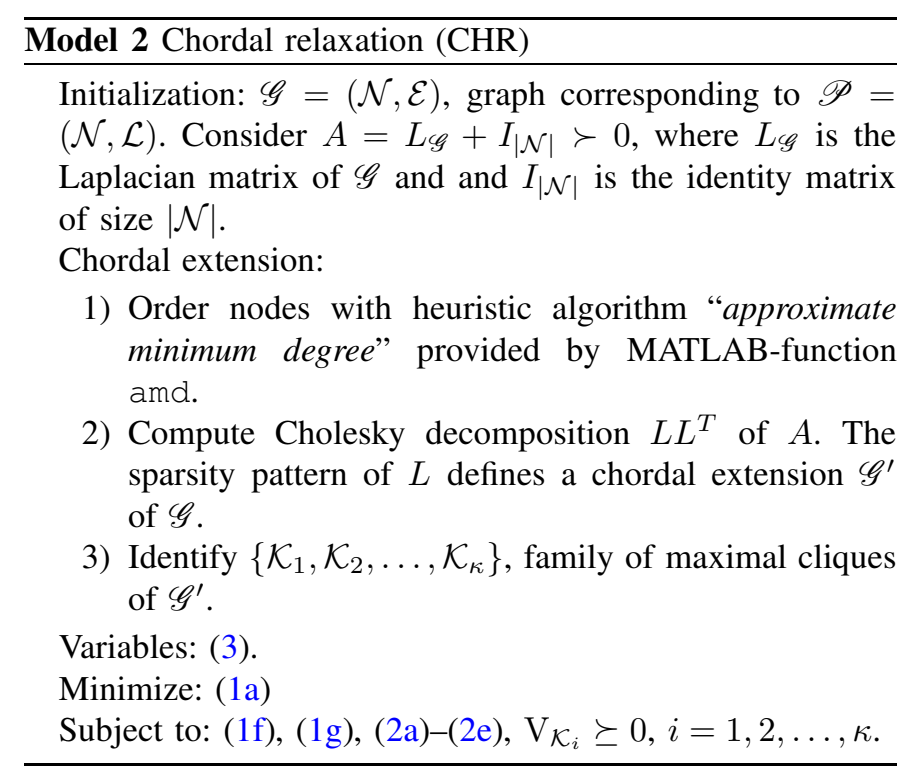

The optimal value $\hat{v}_{C H R}$ of CHR is not affected by the choice of the chordal extension $\mathscr{G}^{\prime}$. However, the optimal choice that minimizes the complexity of CHR is NP-hard to compute. Given a positive definite real matrix $A$ of size $n$ such that $A_{k m}=0$ if $\{k, m\} \notin \mathcal{E}$, let $A=L L^{T}$ be its Cholesky decomposition, where $L$ is a lower triangular matrix. A chordal extension $\mathscr{G}^{\prime}=\left(\mathcal{N}, \mathcal{E}^{\prime}\right)$ of $\mathscr{G}=(\mathcal{N}, \mathcal{E})$ is defined by $\mathcal{E}^{\prime}=\left\{\{k, m\}: L_{k m}+L_{m k} \neq 0, k \neq m\right\}$. The fillin in the Cholesky decomposition depends on the ordering of the nodes $k \in \mathcal{N}$. The problem of finding the ordering that corresponds to the minimum fill-in is known to be NPcomplete. See [12], [23], [24] for more details. Besides, [11], [25]-[27] have developed effective techniques to solve the chordal relaxation of the ACOPF problem and we observe a significant speed-up factor computationally for large-scale power systems compared to the standard SDP relaxation.

\section{Second-order cone relaxation}

If we relax the constraint $\mathrm{V} \succeq 0$ in SDR by $|\mathcal{L}|$ constraints of the form

$$
\mathrm{V}_{\{k, m\}}:=\left[\begin{array}{cc}
\mathrm{V}_{k k} & \mathrm{~V}_{k m} \\
\mathrm{~V}_{k m}^{*} & \mathrm{~V}_{m m}
\end{array}\right] \succeq 0 \forall(k, m) \in \mathcal{L},
$$

we obtain the standard second-order cone relaxation (SOCR) in Model 3. In fact, (4) represents a rotated second-order cone constraint in the $\left(\operatorname{Re}\left(\mathrm{V}_{k m}\right), \operatorname{Im}\left(\mathrm{V}_{k m}\right), \mathrm{V}_{k k}, \mathrm{~V}_{m m}\right)$-space for each branch $(k, m) \in \mathcal{L}$.

Proposition 1 ( [12]). Let $\hat{v}, \hat{v}_{S D R}, \hat{v}_{C H R}, \hat{v}_{S O C R}$ be the optimal values of ACOPF Problem (1), SDR, CHR and SOCR. 


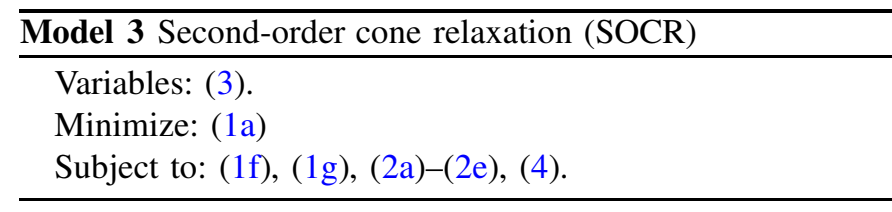

Then $\hat{v}_{S O C R} \leq \hat{v}_{C H R}=\hat{v}_{S D R} \leq \hat{v}$. Moreover, for radial networks, $\hat{v}_{S O C R}=\hat{v}_{C H R}=\hat{v}_{S D R} \leq \hat{v}$.

SOCR is of significant interest because it is computationally more efficient than SDR, and is thus more amenable for largescale instances. It was first proposed in [9] for radial networks, and was extended in [28] to meshed networks by including a trigonometric functional constraint for the voltage angle spread on each line in the network. Later, [19] proposed three strong SOCP relaxations and showed their computational advantages over SDR.

\section{NEW CONIC RELAXATION}

For two real variables $x, y$ such that $\underline{x} \leq x \leq \bar{x}, \underline{y} \leq y \leq \bar{y}$ where $\underline{x}, \bar{x}, \underline{y}, \bar{y} \in \mathbb{R}$ and $\underline{x}<\bar{x}, \underline{y}<\bar{y}$, if $z=x y$ then

$$
\begin{aligned}
& z \leq x \underline{y}+\bar{x} y-\bar{x} \underline{y}, \\
& z \leq x \bar{y}+\underline{x} y-\underline{x} \bar{y}, \\
& z \geq x \underline{y}+\underline{x} y-\underline{x} \underline{y}, \\
& z \geq x \bar{y}+\bar{x} y-\overline{x y} .
\end{aligned}
$$

Inequalities (5) are called reformulation-linearization technique (RLT) inequalities. They describe the convex hull of $\left\{(x, y, z) \in \mathbb{R}^{3}: \underline{x} \leq x \leq \bar{x}, \underline{y} \leq y \leq \bar{y}, z=x y\right\}$ [29]. For a general nonconvex QCQP with bounded real variables, it has been shown in [30] that the use of SDP and RLT constraints together can produce better optimal bounds than either technique used alone. Earlier, it has been proven in [31] that the convex hull of $\left\{(\boldsymbol{x}, X) \in \mathbb{R}^{2} \times \mathbb{H}^{2}: X=\boldsymbol{x} \boldsymbol{x}^{T}, \underline{\boldsymbol{x}} \leq \boldsymbol{x} \leq \overline{\boldsymbol{x}}\right\}$ is given by the SDP constraint $X \succeq \boldsymbol{x} \boldsymbol{x}^{T}$ together with the RLT inequalities on $X_{11}, X_{12}, X_{22}$. Hence, one might be tempted to transform ACOPF Problem (1) with complex variables $\mathbf{v}$ into a problem with real variables $\boldsymbol{v}^{r}:=\operatorname{Re}(\mathbf{v}), \boldsymbol{v}^{i}:=\operatorname{Im}(\mathbf{v})$ and consider a relaxation based on SDP and RLT. Such a relaxation would not be as effective as might be expected due to nonrectangular bounds on $\mathbf{v}$ [32].

On the other hand, it has been shown in [33] that relaxing nonconvex constraints of the ACOPF problem before converting from complex to real variables is more advantageous than doing the operations in opposite order. Thus, assuming $\left|\angle \mathrm{v}_{k}-\angle \mathrm{v}_{m}\right| \leq \pi / 2$ for every branch $(k, m) \in \mathcal{L}$ in the network, equivalent valid inequalities have been proposed in [32], [34] to strengthen the SDP relaxation.

\section{A. Tight-and-cheap relaxation}

For all $(k, m) \in \mathcal{L}$, we have $\mathrm{V}_{k m}=\mathrm{v}_{k} \mathrm{v}_{m}^{*}$ from (2f), therefore $\left|\mathrm{V}_{k m}\right|=\left|\mathrm{v}_{k}\right|\left|\mathrm{v}_{m}\right|$. Considering $x=\left|\mathrm{v}_{k}\right|, y=\left|\mathrm{v}_{m}\right|$,
$z=\left|\mathrm{V}_{k m}\right|$, and applying (5), we obtain

$$
\begin{aligned}
& \left|\mathrm{V}_{k m}\right| \leq\left|\mathrm{v}_{k}\right| \underline{v}_{m}+\bar{v}_{k}\left|\mathrm{v}_{m}\right|-\bar{v}_{k} \underline{v}_{m}, \\
& \left|\mathrm{~V}_{k m}\right| \leq\left|\mathrm{v}_{k}\right| \bar{v}_{m}+\underline{v}_{k}\left|\mathrm{v}_{m}\right|-\underline{v}_{k} \bar{v}_{m}, \\
& \left|\mathrm{~V}_{k m}\right| \geq\left|\mathrm{v}_{k}\right| \underline{v}_{m}+\underline{v}_{k}\left|\mathrm{v}_{m}\right|-\underline{v}_{k} \underline{v}_{m}, \\
& \left|\mathrm{~V}_{k m}\right| \geq\left|\mathrm{v}_{k}\right| \bar{v}_{m}+\bar{v}_{k}\left|\mathrm{v}_{m}\right|-\bar{v}_{k} \bar{v}_{m},
\end{aligned}
$$

since $\bar{v}_{k} \leq\left|\mathrm{v}_{k}\right| \leq \bar{v}_{k}$ for all $k \in \mathcal{N}$. Moreover for all $k \in \mathcal{N}$, $\mathrm{V}_{k k}:=\left|\mathrm{v}_{k}\right|^{2}$, and thus we also have

$$
\mathrm{V}_{k k} \leq\left(\underline{v}_{k}+\bar{v}_{k}\right)\left|\mathrm{v}_{k}\right|-\underline{v}_{k} \bar{v}_{k}
$$

All RLT inequalities (6) are nonconvex, except the constraint (6e) corresponding to the reference bus $k=1$ :

$$
\begin{aligned}
& \operatorname{Re}\left(\mathrm{v}_{1}\right) \geq \frac{\mathrm{V}_{11}+\underline{v}_{1} \bar{v}_{1}}{\underline{v}_{1}+\bar{v}_{1}}, \\
& \operatorname{Im}\left(\mathrm{v}_{1}\right)=0 .
\end{aligned}
$$

Therefore, we define a new formulation of the SDP relaxation in Model 4. We denote nSDR. To the best of our knowledge, it is the first time that nSDR with $\mathrm{V} \succeq \mathbf{v v}^{H}$ is proposed for the ACOPF problem.

\begin{tabular}{l}
\hline Model 4 New semidefinite relaxation (nSDR) \\
\hline Variables: (3), v $\in \mathbb{C}^{|\mathcal{N}|}$. \\
Minimize: (1a) \\
Subject to: (1f), (1g), (2a)-(2e), (7), $\mathrm{V} \succeq \mathbf{v v}^{H}$. \\
\hline
\end{tabular}

Lemma 1. Let $y \in \mathbb{R}$ such that $\ell \leq y \leq u$, where $0 \leq \ell<$ $u<+\infty$. If $x=\sqrt{y}$, then $x \geq \frac{y+\sqrt{\ell} \sqrt{u}}{\sqrt{\ell}+\sqrt{u}}$.

Proof: Let $g(y)=\sqrt{y}$ a concave function on its domain. For all $0 \leq \ell<u<+\infty, \alpha \in[0,1]$,

$$
\begin{aligned}
g((1-\alpha) \ell+\alpha u) & \geq(1-\alpha) g(\ell)+\alpha g(u) \\
& =(1-\alpha) \sqrt{\ell}+\alpha \sqrt{u} .
\end{aligned}
$$

In particular, when $\alpha=\frac{y-\ell}{u-\ell}, \ell \leq y \leq u$, we have

$$
\sqrt{y}=x \geq \frac{y+\sqrt{\ell} \sqrt{u}}{\sqrt{\ell}+\sqrt{u}} .
$$

Lemma 2. Let $\mathrm{A} \in \mathbb{H}^{m}, \mathrm{~B} \in \mathbb{C}^{m \times n}$. If $\mathrm{A} \succeq 0$, then $\mathrm{B}^{H} \mathrm{AB} \succeq 0$.

Proof: Let $\mathbf{x} \in \mathbb{C}^{n}$ and $\mathbf{y}=\mathrm{Bx} \in \mathbb{C}^{m}$. Therefore, $\mathbf{x}^{H} \mathrm{~B}^{H} \mathrm{ABx}=\mathbf{y}^{H} \mathrm{~A} \mathbf{y} \geq 0$.

Proposition 2. $n S D R$ is equivalent to $S D R$.

Proof: Every feasible solution V of nSDR is also feasible for SDR because $\mathrm{V} \succeq \mathbf{v v}^{H} \succeq 0$. It remains to prove that for every feasible solution $\mathrm{V}$ of SDR, there exists $\mathbf{v} \in \mathbb{C}^{n}$ such that $(\mathbf{v}, \mathrm{V})$ is feasible for $\mathrm{nSDR}$.

Given $\mathrm{V}$ feasible solution of SDR, let $\mathbf{v}=\frac{1}{\sqrt{V_{11}}} \mathrm{~V} e_{1}$ where $e_{1}$ is the $n$-dimensional vector with 1 in the first entry and 0 elsewhere. For all $k \in \mathcal{N}, \mathrm{v}_{k}=\frac{1}{\sqrt{V_{11}}} \mathrm{~V}_{k 1}$. In particular, 
$\mathrm{v}_{1}=\sqrt{\mathrm{V}_{11}} \in \mathbb{R}$ and from Lemma 1,

$$
\operatorname{Re}\left(\mathrm{v}_{1}\right)=\sqrt{\mathrm{V}_{11}} \geq \frac{\mathrm{V}_{11}+\underline{v}_{1} \bar{v}_{1}}{\underline{v}_{1}+\bar{v}_{1}} .
$$

Now, let $B=\left[\frac{e_{1}}{\sqrt{V_{11}}} \quad I_{n}\right] \in \mathbb{R}^{n \times(n+1)}$, where $I_{n}$ is the identity matrix of size $n$. From Lemma 2,

$$
B^{T} \mathrm{~V} B=\left[\begin{array}{cc}
1 & \mathbf{v}^{H} \\
\mathbf{v} & \mathrm{V}
\end{array}\right] \succeq 0 \Leftrightarrow \mathrm{V} \succeq \mathbf{v} \mathbf{v}^{H} .
$$

Recall that SOCR is obtained from SDR by replacing the constraint $\mathrm{V} \succeq 0$ in SDR by $|\mathcal{L}|$ smaller positive semidefiniteness constraints, each one corresponding to a branch of the network. Now we replace the constraint $\mathrm{V} \succeq \mathbf{v} \mathbf{v}^{H}$ in Model 4 by $|\mathcal{L}|$ constraints of the form

$$
\left[\begin{array}{ccc}
1 & \mathrm{v}_{k}^{*} & \mathrm{v}_{m}^{*} \\
\mathrm{v}_{k} & \mathrm{~V}_{k k} & \mathrm{~V}_{k m} \\
\mathrm{v}_{m} & \mathrm{~V}_{k m}^{*} & \mathrm{~V}_{m m}
\end{array}\right] \succeq 0 \forall(k, m) \in \mathcal{L} .
$$

to obtain the relaxation given in Model 5. We will refer to this relaxation as "tight-and-cheap relaxation" (TCR). Clearly TCR dominates SOCR and is dominated by SDR.

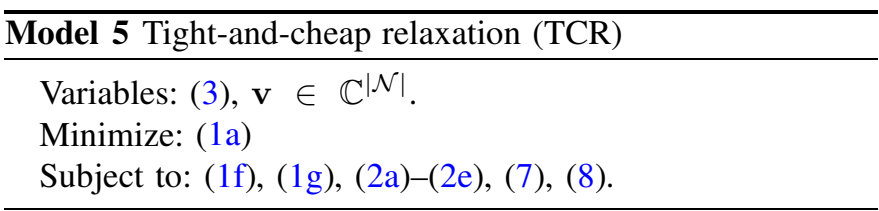

\section{B. Strengthening}

Considering $k=1$ as the reference bus, if the constraint $\mathrm{V} \succeq 0$ in SDR holds, then

$$
\mathrm{V}_{\{1, k, m\}}:=\left[\begin{array}{ccc}
\mathrm{V}_{11} & \mathrm{~V}_{1 k} & \mathrm{~V}_{1 m} \\
\mathrm{~V}_{1 k}^{*} & \mathrm{~V}_{k k} & \mathrm{~V}_{k m} \\
\mathrm{~V}_{1 m}^{*} & \mathrm{~V}_{k m}^{*} & \mathrm{~V}_{m m}
\end{array}\right] \succeq 0 \forall(k, m) \in \mathcal{L} .
$$

We define another relaxation given in Model 6. We call this relaxation "strong tight-and-cheap relaxation" (STCR). Like TCR, STCR dominates SOCR and is dominated by SDR.

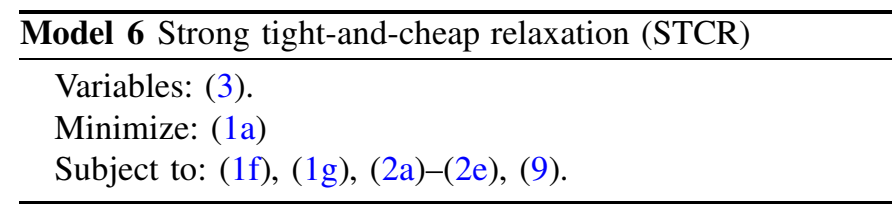

Proposition 3. STCR is stronger than TCR.

Proof: We show that for every $\mathrm{V}$ feasible solution of STCR, there exists $\mathbf{v} \in \mathbb{C}^{n}$ such that $(\mathbf{v}, \mathrm{V})$ is feasible for TCR. For all $k \in \mathcal{N}$, let $\mathrm{v}_{k}=\frac{1}{\sqrt{\mathrm{V}_{11}}} \mathrm{~V}_{k 1}$. In particular, $\mathrm{v}_{1}=$ $\sqrt{\mathrm{V}_{11}} \in \mathbb{R}$ and from Lemma 1 ,

$$
\operatorname{Re}\left(\mathrm{v}_{1}\right)=\sqrt{\mathrm{V}_{11}} \geq \frac{\mathrm{V}_{11}+\underline{v}_{1} \bar{v}_{1}}{\underline{v}_{1}+\bar{v}_{1}} .
$$

Now, for all $(k, m) \in \mathcal{L}$, if (9) holds, then by Lemma 2,

$$
\begin{gathered}
{\left[\begin{array}{cc}
\frac{1}{\sqrt{V_{11}}} & \mathbf{0}^{T} \\
\mathbf{0} & I_{2}
\end{array}\right] \mathrm{V}_{\{1, k, m\}}\left[\begin{array}{cc}
\frac{1}{\sqrt{V_{11}}} & \mathbf{0}^{T} \\
\mathbf{0} & I_{2}
\end{array}\right]} \\
=\left[\begin{array}{ccc}
1 & \mathrm{v}_{k}^{*} & \mathrm{v}_{m}^{*} \\
\mathrm{v}_{k} & \mathrm{~V}_{k k} & \mathrm{~V}_{k m} \\
\mathrm{v}_{m} & \mathrm{~V}_{k m}^{*} & \mathrm{~V}_{m m}
\end{array}\right] \succeq 0 .
\end{gathered}
$$

Proposition 4. Let $\mathscr{G}=(\mathcal{N}, \mathcal{E})$ a graph corresponding to a power network $\mathscr{P}=(\mathcal{N}, \mathcal{L})$. Suppose bus $k=1$ is the reference bus. If the induced subgraph $\mathscr{G}-\{1\}$ has no cycle, then STCR is equivalent to SDR.

Proof: Consider $\mathscr{G}^{\prime}=\left(\mathcal{N}, \mathcal{E}^{\prime}\right)$ where $\mathcal{E}^{\prime}=\mathcal{E} \cup$ $\{\{1, m\}:\{1, m\} \notin \mathcal{E}\}$. Since $\mathscr{G}-\{1\}$ has no cycle, every cycle in $\mathscr{G}^{\prime}$ contains vertex $k=1$. Also, since for all $m \in \mathcal{N} \backslash\{1\},\{1, m\} \in \mathcal{E}^{\prime}$, every cycle $1-m_{1}-m_{2}-m_{3}-1$ of 4 vertices has a chord $\left\{1, m_{2}\right\}$. Thus, $\mathscr{G}^{\prime}$ is a chordal extension of $\mathscr{G}$.

On the other hand, since $\mathscr{G}-\{1\}$ has no cycle, every maximal clique $\mathcal{K}$ of $\mathscr{G}^{\prime}$ contains vertex $k=1$ and has at most 3 vertices, i.e. $\mathcal{K}=\{1, k, m\}$ for all $\{k, m\} \in \mathcal{E}$. Then the constraint $\mathrm{V} \succeq 0$ in SDR is equivalent to $\mathrm{V}_{\{1, k, m\}} \succeq 0$ for all $\{k, m\} \in \mathcal{E}$.

\section{Computational Results}

In this section, we evaluate the accuracy and the computational efficiency of TCR and STCR as compared to SOCR, CHR and SDR.

We tested the models $1,2,3,5$ and 6 on standard test cases available from MATPOWER [20], [35], [36]. It is important to note that, unlike what was done in [21], [25]-[27], we did not make any modification or simplification to the data.

We solved all the relaxations in MATLAB using CVX 2.1 [37], [38] with the solver MOSEK 8.0.0.60 and default precision (tolerance $\epsilon=1.49 \times 10^{-8}$ ). All the computations were carried out on an Intel Core i7-6700 CPU \& $3.40 \mathrm{GHz}$ computing platform. When solving SOCR for instances with at least 1000 buses, MOSEK ended its computation with message Mosek error: MSK_RES_TRM_STALL(). For these test cases, we replace constraints (4) by equivalent ones

$$
\left[\begin{array}{ccc}
\mathrm{V}_{k k}+\mathrm{V}_{m m} & 0 & 2 \mathrm{~V}_{k m} \\
0 & \mathrm{~V}_{k k}+\mathrm{V}_{m m} & \mathrm{~V}_{k k}-\mathrm{V}_{m m} \\
2 \mathrm{~V}_{k m}^{*} & \mathrm{~V}_{k k}-\mathrm{V}_{m m} & \mathrm{~V}_{k k}+\mathrm{V}_{m m}
\end{array}\right] \succeq 0
$$

for all $(k, m) \in \mathcal{L}$.

We considered two objective functions: the generation cost $[\$ / \mathrm{h}](1 \mathrm{a})$ and the active loss [MW] where $c_{g 2}=0, c_{g 1}=$ 1 and $c_{g 0}=0$ for all $g \in \mathcal{G}$ in (1a). Both objective functions of test cases from [36] are the same. We denote $\underline{v}$ the best lower bound which is the maximum value among $\hat{v}_{S O C R}, \hat{v}_{T C R}$, $\hat{v}_{S T C R}, \hat{v}_{C H R}, \hat{v}_{S D R}$, respective optimal values of SOCR, TCR, STCR, CHR and SDR. The optimality gap is measured as $100\left(1-\hat{v}_{R} / \bar{v}\right)$, where $\bar{v}$ is the upper bound provided by the MATPOWER-solver "MIPS" and $\hat{v}_{R}$ is the relaxation optimal value. For some test cases: 1888 rte, 1951 rte, 2848 rte, 
2868rte, 6468rte, 6470rte, 6495rte and 6515rte, MIPS failed to find a local optimal solution, so we considered the upper bounds reported in [36].

Table I and Table II summarize the optimality gaps of the five relaxations for cost minimization and loss minimization, respectively. The results support the following key points:

1) CHR is equivalent to SDR as predicted by Proposition 1.

2) TCR and STCR are stronger than SOCR. When compared to SOCR, TCR reduces the optimality gap from $0.17 \%$ to $0.06 \%$ on average for large-scale instances in Table II.

3) Optimality gaps of TCR and STCR are very close to CHR or SDR. We observe significant optimality gaps of TCR and STCR when the optimality gap of CHR or SDR is not close to zero, e.g. case 5 and case_ACTIV_SG_500 instances in Table I.

4) STCR is stronger than TCR as predicted by Proposition 3. For example, STCR reduces substantially the optimality gap of TCR from $12.75 \%$ to $5.22 \%$ on case 5 instance in Table I.

The computation times reported by MOSEK are shown in Table I and Table II. The time CVX took to pre-compile a model is not included. For CHR, the computation time does not take into account the time of building the chordal extension of an instance's graph. We did not solve SDR for the extra large-scale instances (those with at least 6000 buses) because of the high computational cost. In Tables I and II, we note:

1) Among all relaxations, SOCR is the fastest and SDR is the slowest.

2) $\mathrm{CHR}$ is on average around 30 times faster than SDR for large-scale instances.

3) TCR is on average around 30 times faster than CHR for large-scale instances and 55 times for extra large-scale instances.

4) TCR is on average around 3 times faster than STCR for large-scale instances and 7 times for extra large-scale instances.

\section{CONCLUSION}

We proposed a new formulation of the semidefinite relaxation for the ACOPF problem. This formulation is based on a positive semidefiniteness constraint combined with reformulation-linearization technique (RLT) constraints defined on the reference (slack) bus. We proved that it is equivalent to the standard SDP relaxation. Thereafter, we derived a tight-and-cheap semidefinite relaxation (TCR) stronger than the standard SOCP relaxation. Experiments on unmodified MATPOWER instances show that the proposed relaxation offers an interesting trade-off between the standard SDP and SOCP relaxations for large-scale power systems because it is very close to the SDP relaxation in terms of optimality gap, but computationally it is much faster than the chordal relaxation (which is equivalent to the SDP relaxation).

A strong TCR (STCR) was also proposed. We showed that, under some assumption, it is tantamount to the standard SDP relaxation. Although faster than the chordal relaxation, it is not as fast as TCR.

\section{ACKNOWLEDGMENT}

We thank Stéphane Alarie and Laurent Lenoir, both of the Hydro-Québec Research Institute (IREQ), for helpful comments on early drafts of this paper. We also thank the anonymous reviewers for their many helpful suggestions that helped us improve this paper.

\section{REFERENCES}

[1] J. Carpentier. Contribution to the economic dispatch problem. Bulletin de la Société française des électriciens, 3(8):431-447, 1962.

[2] Mary B. Cain, Richard P. ONeill, and Anya Castillo. History of optimal power flow and formulations. Federal Energy Regulatory Commission, pages 1-36, 2012.

[3] Stephen Frank, Ingrida Steponavice, and Steffen Rebennack. Optimal power flow: A bibliographic survey I. Energy Systems, 3(3):221-258, 2012.

[4] Stephen Frank, Ingrida Steponavice, and Steffen Rebennack. Optimal power flow: A bibliographic survey II. Energy Systems, 3(3):259-289, 2012.

[5] Abhinav Verma. Power Grid Security Analysis: An Optimization Approach. Columbia University, 2010.

[6] Karsten Lehmann, Alban Grastien, and Pascal Van Hentenryck. ACfeasibility on tree networks is NP-hard. IEEE Transactions on Power Systems, 31(1):798-801, 2016.

[7] Stephen Boyd and Lieven Vandenberghe. Convex Optimization. Cambridge University Press, 2004.

[8] Christoph Helmberg. Semidefinite Programming for Combinatorial Optimization. Konrad-Zuse-Zentrum für Informationstechnik Berlin, 2000.

[9] R. A. Jabr. Radial distribution load flow using conic programming. IEEE Transactions on Power Systems, 21(3):1458-1459, Aug 2006.

[10] Xiaoqing Bai, Hua Wei, Katsuki Fujisawa, and Yong Wang. Semidefinite programming for optimal power flow problems. International Journal of Electrical Power \& Energy Systems, 30(6):383-392, 2008.

[11] R. A. Jabr. Exploiting sparsity in SDP relaxations of the OPF problem. IEEE Transactions on Power Systems, 27(2):1138-1139, May 2012.

[12] S. H. Low. Convex relaxation of optimal power flow-Part I: Formulations and equivalence. IEEE Transactions on Control of Network Systems, 1(1):15-27, March 2014.

[13] S. H. Low. Convex relaxation of optimal power flow-Part II: Exactness. IEEE Transactions on Control of Network Systems, 1(2):177-189, June 2014.

[14] Hassan Hijazi, Carleton Coffrin, and Pascal Van Hentenryck. Convex quadratic relaxations for mixed-integer nonlinear programs in power systems. Mathematical Programming Computation, pages 1-47, 2014.

[15] Hassan Hijazi, Carleton Coffrin, and Pascal Van Hentenryck. Polynomial SDP cuts for optimal power flow. In Power Systems Computation Conference (PSCC), 2016, pages 1-7. IEEE, 2016.

[16] Cédric Josz, Jean Maeght, Patrick Panciatici, and Jean Charles Gilbert. Application of the moment-SOS approach to global optimization of the OPF problem. IEEE Transactions on Power Systems, 30(1):463-470, 2015.

[17] Carleton Coffrin, Hassan Hijazi, and Pascal Van Hentenryck. Network flow and copper plate relaxations for AC transmission systems. In Power Systems Computation Conference (PSCC), 2016, pages 1-8. IEEE, 2016.

[18] Sleiman Mhanna, Gregor Verbič, and Archie C. Chapman. Tight LP approximations for the optimal power flow problem. In Power Systems Computation Conference (PSCC), 2016, pages 1-7. IEEE, 2016.

[19] Burak Kocuk, Santanu S. Dey, and X. Andy Sun. Strong SOCP relaxations for the optimal power flow problem. Operations Research, 64(6):1177-1196, 2016.

[20] R. D. Zimmerman, C. E. Murillo-Sanchez, and R. J. Thomas. MATPOWER: Steady-state operations, planning, and analysis tools for power systems research and education. IEEE Transactions on Power Systems, 26(1):12-19, Feb 2011.

[21] Javad Lavaei and Steven H. Low. Zero duality gap in optimal power flow problem. IEEE Transactions on Power Systems, 27(1):92-107, 2012.

[22] Robert Grone, Charles R. Johnson, Eduardo M. Sá, and Henry Wolkowicz. Positive definite completions of partial Hermitian matrices. Linear algebra and its applications, 58:109-124, 1984.

[23] Mituhiro Fukuda, Masakazu Kojima, Kazuo Murota, and Kazuhide Nakata. Exploiting sparsity in semidefinite programming via matrix completion I: General framework. SIAM Journal on Optimization, 11(3):647-674, 2001. 
TABLE I: Cost minimization

\begin{tabular}{|c|c|c|c|c|c|c|c|c|c|c|c|c|}
\hline \multirow[t]{2}{*}{ Test case } & \multirow[t]{2}{*}{$\bar{v}[\$ / h]$} & \multirow[t]{2}{*}{$\underline{v}[\$ / h]$} & \multicolumn{5}{|c|}{ Optimality gap [\%] } & \multicolumn{5}{|c|}{ Computation time $[\mathrm{s}]$} \\
\hline & & & SOCR & TCR & STCR & CHR & SDR & SOCR & TCR & STCR & CHR & $\overline{S D R}$ \\
\hline \multicolumn{13}{|c|}{ Small-scale instances } \\
\hline LMBD 3_50 & 5812.64 & 5789.91 & 1.32 & 0.74 & 0.39 & 0.39 & 0.39 & 0.08 & 0.06 & 0.06 & 0.06 & 0.06 \\
\hline LMBD 3_60 & 5707.11 & 5707.11 & 0.05 & 0.00 & 0.00 & 0.00 & 0.00 & 0.07 & 0.08 & 0.06 & 0.06 & 0.05 \\
\hline case 5 & 17551.89 & 16635.78 & 14.54 & 12.75 & 5.22 & 5.22 & 5.22 & 0.06 & 0.07 & 0.07 & 0.07 & 0.07 \\
\hline case 6 ww & 3143.97 & 3143.97 & 0.63 & 0.00 & 0.00 & 0.00 & 0.00 & 0.09 & 0.07 & 0.07 & 0.07 & 0.06 \\
\hline case 9 & 5296.69 & 5296.69 & 0.00 & 0.00 & 0.00 & 0.00 & 0.00 & 0.07 & 0.07 & 0.07 & 0.07 & 0.07 \\
\hline case14 & 8081.53 & 8081.52 & 0.08 & 0.00 & 0.00 & 0.00 & 0.00 & 0.07 & 0.08 & 0.07 & 0.08 & 0.07 \\
\hline case24_ieee_rts & 63352.21 & 63352.20 & 0.01 & 0.00 & 0.00 & 0.00 & 0.00 & 0.09 & 0.13 & 0.14 & 0.11 & 0.13 \\
\hline case 30 & 576.89 & 576.89 & 0.57 & 0.07 & 0.00 & 0.00 & 0.00 & 0.08 & 0.14 & 0.16 & 0.13 & 0.16 \\
\hline case_ieee 30 & 8906.14 & 8906.14 & 0.04 & 0.00 & 0.00 & 0.00 & 0.00 & 0.08 & 0.11 & 0.11 & 0.08 & 0.10 \\
\hline case 39 & 41864.18 & 41862.03 & 0.02 & 0.01 & 0.01 & 0.01 & 0.01 & 0.08 & 0.19 & 0.18 & 0.11 & 0.25 \\
\hline case 57 & 41737.79 & 41737.78 & 0.06 & 0.01 & 0.00 & 0.00 & 0.00 & 0.08 & 0.17 & 0.19 & 0.16 & 0.25 \\
\hline case89pegase & 5819.81 & 5819.65 & 0.17 & 0.04 & 0.00 & 0.00 & 0.00 & 0.16 & 0.68 & 0.83 & 0.90 & 1.08 \\
\hline Average & & & 0.39 & 0.23 & 0.09 & 0.09 & 0.09 & 0.10 & 0.30 & 0.34 & $\mathbf{0 . 3 4}$ & 0.43 \\
\hline \multicolumn{13}{|c|}{ Medium-scale instances } \\
\hline case118 & 129660.70 & 129654.54 & 0.25 & 0.03 & 0.02 & 0.00 & 0.00 & 0.08 & 0.33 & 0.42 & 0.32 & $\overline{1.09}$ \\
\hline case_ACTIV_SG_200 & 27557.57 & 27557.55 & 0.00 & 0.00 & 0.00 & 0.00 & 0.00 & 0.21 & 0.56 & 0.65 & 0.72 & 4.66 \\
\hline case_illinois 200 & 36748.39 & 36748.33 & 0.02 & 0.00 & 0.00 & 0.00 & 0.00 & 0.22 & 0.83 & 0.92 & 0.87 & 6.28 \\
\hline case $\overline{3} 00$ & 719725.11 & 719710.63 & 0.15 & 0.02 & 0.01 & 0.00 & 0.00 & 0.20 & 0.97 & 1.20 & 0.95 & 10.52 \\
\hline case_ACTIV_SG_500 & 72578.30 & 71048.04 & 5.38 & 4.39 & 4.20 & 2.11 & 2.11 & 0.75 & 3.21 & 4.04 & 3.14 & 103.35 \\
\hline Average & & & 2.10 & 1.67 & 1.60 & 0.80 & 0.80 & 0.40 & 1.68 & 2.08 & 1.68 & 43.36 \\
\hline \multicolumn{13}{|c|}{ Large-scale instances } \\
\hline case1354pegase & 74069.35 & 74061.72 & 0.08 & 0.02 & 0.02 & 0.01 & 0.01 & 5.73 & 6.34 & 13.23 & 9.72 & 1657.85 \\
\hline case1888rte & 59805.1 & 59601.29 & 0.39 & 0.36 & 0.35 & 0.34 & 0.34 & 8.88 & 10.61 & 26.64 & 17.88 & 4629.31 \\
\hline case1951rte & 81737.7 & 81725.16 & 0.08 & 0.03 & 0.03 & 0.01 & 0.02 & 11.24 & 12.49 & 31.78 & 23.31 & 5595.59 \\
\hline case2383wp & 1868511.83 & 1861214.70 & 1.07 & 0.50 & 0.48 & 0.40 & 0.39 & 11.55 & 11.60 & 33.76 & 216.38 & 9420.67 \\
\hline case2736sp & 1307883.13 & 1307695.31 & 0.31 & 0.03 & 0.01 & 0.02 & 0.02 & 10.24 & 12.28 & 36.42 & 303.63 & 10786.69 \\
\hline case2737sop & 777629.30 & 777517.52 & 0.27 & 0.03 & 0.02 & 0.02 & 0.01 & 8.90 & 11.47 & 35.68 & 242.92 & 10601.37 \\
\hline case 2746 wop & 1208279.81 & 1208182.18 & 0.40 & 0.03 & 0.02 & 0.01 & 0.01 & 9.87 & 11.51 & 35.57 & 343.90 & 10480.83 \\
\hline case2746wp & 1631775.10 & 1631665.81 & 0.33 & 0.03 & 0.02 & 0.01 & 0.01 & 11.34 & 13.00 & 36.68 & 340.18 & 11654.59 \\
\hline case2848rte & 53021.8 & 53005.20 & 0.08 & 0.04 & 0.04 & 0.03 & 0.04 & 12.17 & 14.36 & 44.56 & 46.64 & 14567.98 \\
\hline case2868rte & 79794.7 & 79787.67 & 0.07 & 0.02 & 0.02 & 0.01 & 0.02 & 14.05 & 17.03 & 55.08 & 47.20 & 16933.60 \\
\hline case2869pegase & 133999.29 & 133983.11 & 0.09 & 0.03 & 0.03 & 0.01 & 0.03 & 15.45 & 18.76 & 60.18 & 49.29 & 14866.00 \\
\hline case3012wp & 2591706.57 & 2587512.73 & 0.82 & 0.38 & 0.37 & 0.17 & 0.16 & 12.90 & 15.21 & 49.39 & 1276.67 & 16051.95 \\
\hline case 3120 sp & 2142703.76 & 2140385.00 & 0.56 & 0.13 & 0.12 & 0.11 & 0.11 & 14.98 & 16.55 & 52.77 & 765.39 & 14636.04 \\
\hline case 3375 wp & 7412030.68 & 7407116.46 & 0.30 & 0.14 & 0.14 & 0.08 & 0.07 & 15.23 & 17.64 & 66.37 & 1216.15 & 18229.90 \\
\hline Average & & & 0.36 & 0.12 & 0.12 & 0.08 & 0.08 & 12.03 & 14.02 & 43.71 & 402.09 & 12336.09 \\
\hline \multicolumn{13}{|c|}{ Extra large-scale instances } \\
\hline case6468rte & 86860.0 & 86808.12 & 0.27 & 0.08 & 0.08 & 0.06 & - & 35.47 & 40.79 & 314.92 & 1990.53 & - \\
\hline case6470rte & 98345.5 & 98333.90 & 0.18 & 0.06 & 0.03 & 0.01 & - & 53.34 & 51.88 & 371.42 & 2729.29 & - \\
\hline case6495rte & 106283.4 & $106 \quad 072.44$ & 0.46 & 0.23 & 0.23 & 0.20 & - & 50.50 & 65.15 & 431.65 & 3592.15 & - \\
\hline case 6515 rte & 109804.2 & 109688.71 & 0.38 & 0.16 & 0.14 & 0.11 & - & 48.02 & 59.60 & 427.22 & 3523.01 & - \\
\hline Average & & & 0.32 & 0.13 & 0.12 & 0.09 & - & 46.84 & 54.38 & 386.42 & 2960.41 & . \\
\hline
\end{tabular}

[24] Kazuhide Nakata, Katsuki Fujisawa, Mituhiro Fukuda, Masakazu Kojima, and Kazuo Murota. Exploiting sparsity in semidefinite programming via matrix completion II: Implementation and numerical results. Mathematical Programming, 95(2):303-327, 2003.

[25] Daniel K. Molzahn, Jesse T. Holzer, Bernard C. Lesieutre, and Christopher L. DeMarco. Implementation of a large-scale optimal power flow solver based on semidefinite programming. IEEE Transactions on Power Systems, 28(4):3987-3998, 2013.

[26] Martin S. Andersen, Anders Hansson, and Lieven Vandenberghe. Reduced-complexity semidefinite relaxations of optimal power flow problems. IEEE Transactions on Power Systems, 29(4):1855-1863, 2014.

[27] R. Madani, M. Ashraphijuo, and J. Lavaei. OPF solver. https://lavaei.ieor.berkeley.edu/Software.html, 2014.

[28] R. A. Jabr. A conic quadratic format for the load flow equations of meshed networks. IEEE Transactions on Power Systems, 22(4):22852286, Nov 2007.

[29] Garth P. McCormick. Computability of global solutions to factorable nonconvex programs-Part I: Convex underestimating problems. Mathematical Programming, 10(1):147-175, 1976.

[30] Kurt M. Anstreicher. Semidefinite programming versus the reformulation-linearization technique for nonconvex quadratically constrained quadratic programming. Journal of Global Optimization, 43(2-
3):471-484, 2009

[31] Kurt M. Anstreicher and Samuel Burer. Computable representations for convex hulls of low-dimensional quadratic forms. Mathematical programming, 124(1):33-43, 2010.

[32] Chen Chen, Alper Atamtürk, and Shmuel S. Oren. A spatial branchand-cut method for nonconvex QCQP with bounded complex variables. Mathematical Programming, pages 1-29, 2017.

[33] Cédric Josz and Daniel K. Molzahn. Moment/sum-of-squares hierarchy for complex polynomial optimization. arXiv preprint arXiv:1508.02068, 2015.

[34] Carleton Coffrin, Hassan L. Hijazi, and Pascal Van Hentenryck Strengthening the SDP relaxation of AC power flows with convex envelopes, bound tightening, and valid inequalities. IEEE Transactions on Power Systems, 32(5):3549-3558, 2017.

[35] A. B. Birchfield, T. Xu, K. M. Gegner, K. S. Shetye, and T. J. Overbye. Grid structural characteristics as validation criteria for synthetic networks. IEEE Transactions on Power Systems, 32(4):3258-3265, July 2017.

[36] Cédric Josz, Stéphane Fliscounakis, Jean Maeght, and Patrick Panciatici. AC power flow data in MATPOWER and QCQP format: iTesla, RTE snapshots, and PEGASE. arXiv preprint arXiv:1603.01533, 2016.

[37] CVX Research, Inc. CVX: MATLAB software for disciplined convex programming, version 2.1. http://cvxr.com/cvx, August 2012. 
TABLE II: Loss minimization

\begin{tabular}{|c|c|c|c|c|c|c|c|c|c|c|c|c|}
\hline \multirow[t]{2}{*}{ Test case } & \multirow[t]{2}{*}{$\bar{v}[\mathrm{MW}]$} & \multirow[t]{2}{*}{$\underline{v}[\mathrm{MW}]$} & \multicolumn{5}{|c|}{ Optimality gap [\%] } & \multicolumn{5}{|c|}{ Computation time $[\mathrm{s}]$} \\
\hline & & & SOCR & TCR & STCR & CHR & SDR & SOCR & TCR & STCR & CHR & SDR \\
\hline \multicolumn{13}{|c|}{ Small-scale instances } \\
\hline LMBD 3_50 & 317.38 & 317.38 & 0.00 & 0.00 & 0.00 & 0.00 & 0.00 & 0.05 & 0.06 & 0.06 & 0.05 & 0.06 \\
\hline LMBD 3_60 & 316.75 & 316.75 & 0.01 & 0.00 & 0.00 & 0.00 & 0.00 & 0.05 & 0.05 & 0.07 & 0.05 & 0.06 \\
\hline case 5 & 1001.06 & 1001.06 & 0.00 & 0.00 & 0.00 & 0.00 & 0.00 & 0.08 & 0.10 & 0.09 & 0.09 & 0.09 \\
\hline case 6 ww & 216.84 & 216.84 & 0.16 & 0.00 & 0.00 & 0.00 & 0.00 & 0.06 & 0.07 & 0.06 & 0.08 & 0.06 \\
\hline case 9 & 317.32 & 317.32 & 0.00 & 0.00 & 0.00 & 0.00 & 0.00 & 0.07 & 0.07 & 0.07 & 0.07 & 0.07 \\
\hline case14 & 259.55 & 259.55 & 0.00 & 0.00 & 0.00 & 0.00 & 0.00 & 0.07 & 0.07 & 0.07 & 0.06 & 0.05 \\
\hline case24_ieee_rts & 2875.75 & 2875.74 & 0.01 & 0.00 & 0.00 & 0.00 & 0.00 & 0.10 & 0.14 & 0.14 & 0.12 & 0.16 \\
\hline case 30 & 191.09 & 191.09 & 0.23 & 0.01 & 0.00 & 0.00 & 0.00 & 0.08 & 0.13 & 0.15 & 0.10 & 0.18 \\
\hline case_ieee 30 & 284.77 & 284.77 & 0.05 & 0.00 & 0.00 & 0.00 & 0.00 & 0.07 & 0.09 & 0.09 & 0.08 & 0.27 \\
\hline case 39 & 6284.15 & 6283.90 & 0.01 & 0.00 & 0.00 & 0.00 & 0.00 & 0.09 & 0.19 & 0.18 & 0.12 & 0.22 \\
\hline case 57 & 1262.10 & 1262.10 & 0.03 & 0.00 & 0.00 & 0.00 & 0.00 & 0.08 & 0.14 & 0.16 & 0.15 & 0.21 \\
\hline case89pegase & 5819.81 & 5819.65 & 0.17 & 0.04 & 0.00 & 0.00 & 0.00 & 0.16 & 0.68 & 0.83 & 0.90 & 1.08 \\
\hline Average & & & 0.09 & 0.01 & 0.00 & 0.00 & $\mathbf{0 . 0 0}$ & 0.10 & 0.29 & $\mathbf{0 . 3 3}$ & 0.34 & 0.44 \\
\hline \multicolumn{13}{|c|}{ Medium-scale instances } \\
\hline case118 & 4251.23 & 4251.03 & 0.01 & 0.01 & 0.00 & 0.00 & 0.00 & 0.09 & 0.31 & 0.35 & 0.25 & 0.83 \\
\hline case_ACTIV_SG_200 & 1483.92 & 1483.92 & 0.01 & 0.00 & 0.00 & 0.00 & 0.00 & 0.19 & 0.52 & 0.65 & 0.56 & 4.07 \\
\hline case_illinois 200 & 2246.49 & 2246.48 & 0.01 & 0.00 & 0.00 & 0.00 & 0.00 & 0.25 & 0.79 & 0.90 & 0.83 & 5.80 \\
\hline case 300 & 23737.72 & 23737.55 & 0.06 & 0.01 & 0.01 & 0.00 & 0.00 & 0.19 & 0.91 & 1.05 & 0.86 & 9.93 \\
\hline case_ACTIV_SG_500 & 7817.46 & 7817.41 & 0.02 & 0.00 & 0.00 & 0.00 & 0.00 & 0.75 & 4.04 & 5.03 & 3.75 & 127.07 \\
\hline Average & & & 0.02 & 0.00 & $\mathbf{0 . 0 0}$ & 0.00 & $\mathbf{0 . 0 0}$ & 0.40 & 1.97 & 2.41 & 1.85 & 52.04 \\
\hline \multicolumn{13}{|c|}{ Large-scale instances } \\
\hline case1354pegase & 74069.35 & 74061.72 & 0.08 & 0.02 & 0.02 & 0.01 & 0.01 & 5.73 & 6.34 & 13.23 & 9.72 & 1657.85 \\
\hline case1888rte & 59805.1 & 59601.29 & 0.39 & 0.36 & 0.35 & 0.34 & 0.34 & 8.88 & 10.61 & 26.64 & 17.88 & 4629.31 \\
\hline case1951rte & 81737.7 & 81725.16 & 0.08 & 0.03 & 0.03 & 0.01 & 0.02 & 11.24 & 12.49 & 31.78 & 23.31 & 5595.59 \\
\hline case2383wp & 24991.40 & 24979.30 & 0.21 & 0.07 & 0.07 & 0.05 & 0.05 & 10.83 & 10.88 & 32.25 & 188.90 & 7758.35 \\
\hline case2736sp & 18335.95 & 18334.71 & 0.19 & 0.03 & 0.02 & 0.01 & 0.01 & 9.27 & 11.01 & 31.96 & 290.60 & 10768.88 \\
\hline case2737sop & 11397.39 & 11396.61 & 0.18 & 0.02 & 0.01 & 0.01 & 0.01 & 8.46 & 11.38 & 29.65 & 216.13 & 9715.82 \\
\hline case 2746 wop & 19212.35 & 19211.42 & 0.21 & 0.03 & 0.02 & 0.01 & 0.00 & 10.15 & 11.26 & 30.92 & 320.35 & 9093.47 \\
\hline case2746wp & 25269.45 & 25268.43 & 0.19 & 0.03 & 0.01 & 0.00 & 0.01 & 10.76 & 12.34 & 33.78 & 336.99 & 11785.35 \\
\hline case 2848 rte & 53021.8 & 53005.20 & 0.08 & 0.04 & 0.04 & 0.03 & 0.04 & 12.17 & 14.36 & 44.56 & 46.64 & 14567.98 \\
\hline case2868rte & 79794.7 & 79787.67 & 0.07 & 0.02 & 0.02 & 0.01 & 0.02 & 14.05 & 17.03 & 55.08 & 47.20 & 16933.60 \\
\hline case2869pegase & 133999.29 & 133983.11 & 0.09 & 0.03 & 0.03 & 0.01 & 0.03 & 15.45 & 18.76 & 60.18 & 49.29 & 14866.00 \\
\hline case3012wp & 27645.97 & 27637.25 & 0.22 & 0.05 & 0.04 & 0.04 & 0.03 & 16.80 & 17.57 & 55.82 & 1448.81 & 17689.12 \\
\hline case3120sp & 21513.52 & 21495.85 & 0.24 & 0.10 & 0.09 & 0.09 & 0.08 & 15.08 & 17.09 & 58.92 & 830.41 & 15981.61 \\
\hline case $3375 w p$ & 49004.69 & 48995.70 & 0.15 & 0.04 & 0.03 & 0.02 & 0.03 & 15.07 & 16.85 & 70.58 & 1229.45 & 17306.68 \\
\hline Average & & & 0.17 & 0.06 & 0.05 & 0.04 & 0.04 & 12.17 & 13.97 & 43.70 & 416.32 & 12230.42 \\
\hline \multicolumn{13}{|c|}{ Extra large-scale instances } \\
\hline case6468rte & 86860.0 & 86808.12 & 0.27 & 0.08 & 0.08 & 0.06 & - & 35.47 & 40.79 & 314.92 & 1990.53 & 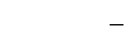 \\
\hline case6470rte & 98345.5 & 98333.90 & 0.18 & 0.06 & 0.03 & 0.01 & - & 53.34 & 51.88 & 371.42 & 2729.29 & - \\
\hline case6495rte & 106283.4 & 106072.44 & 0.46 & 0.23 & 0.23 & 0.20 & - & 50.50 & 65.15 & 431.65 & 3592.15 & - \\
\hline case6515rte & 109804.2 & 109688.71 & 0.38 & 0.16 & 0.14 & 0.11 & - & 48.02 & 59.60 & 427.22 & 3523.01 & 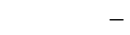 \\
\hline Average & & & 0.32 & 0.13 & 0.12 & 0.09 & - & 46.84 & 54.38 & 386.42 & 2960.41 & - \\
\hline
\end{tabular}

[38] M. Grant and S. Boyd. Graph implementations for nonsmooth convex programs. In V. Blondel, S. Boyd, and H. Kimura, editors, Recent Advances in Learning and Control, Lecture Notes in Control and Information Sciences, pages 95-110. Springer-Verlag Limited, 2008. http://stanford.edu/ boyd/graph_dcp.html.

Christian Bingane (S'18) received the B.Eng. degree in electrical engineering in 2014 from Polytechnique Montreal, Montreal, QC, Canada, where he is currently working toward the Ph.D. degree in applied mathematics. He is currently a student member of the GERAD research center.

His research interests include optimization in power systems and conic programming. He is concerned with using linear programming, second-order cone programming or semidefinite programming to provide guaranteed global optimal solution to the optimal power flow problem for a large-scale power system.
Miguel F. Anjos (M'07-SM'18) received the B.Sc. degree, the M.S. degree and the Ph.D. degree from McGill University, Montreal, QC, Canada, Stanford University, Stanford, CA, USA, and the University of Waterloo, Waterloo, ON, Canada in 1992, 1994 and 2001 respectively.

$\mathrm{He}$ is currently a Professor with the Department of Mathematics and Industrial Engineering, Polytechnique Montreal, Montreal, QC, Canada, where he holds the NSERC-Hydro-Quebec-Schneider Electric Industrial Research Chair, and an Inria International Chair. He is a Licensed Professional Engineer in Ontario, Canada. He served for five years as Editor-in-Chief of Optimization and Engineering, and serves on several editorial boards.

His allocades include a Canada Research Chair, the Méritas Teaching Award, a Humboldt Research Fellowship, the title of EUROPT Fellow, and the Queen Elizabeth II Diamond Jubilee Medal. He is an elected Fellow of the Canadian Academy of Engineering.

Sébastien Le Digabel received the M.Sc.A. degree and the Ph.D. degree in applied mathematics from Polytechnique Montreal, Montreal, Quebec, Canada in 2002 and 2008 respectively. He was a postdoctoral fellow with the IBM Watson Research Center and the University of Chicago in 2010 and 2011. 
He is currently an Associate Professor with the Department of Mathematics and Industrial Engineering, Polytechnique Montreal, Montreal, QC, Canada, and a regular member of the GERAD research center.

His research interests include the analysis and development of algorithms for derivative-free and blackbox optimization, and the design of related software. All of his work on derivative-free optimization is included in the NOMAD software, a free package for blackbox optimization available at www.gerad.ca/nomad. 